correlated with reference to measured iron levels in the surrounding brain tissue and the extent of erythrolysis/hemolysis within the hematoma.

Results Iron overload (IO) to the brain tissue surrounding the hematoma from a given hematoma correlates positively with increasing size of the hematoma. The erythrolysis/hemolysis proportion increases significantly with time up to day 14 , evident in almost each individual patient. The IO also positively correlates with proportion of early erythrolysis/hemolysis at days $14 \& 30$. Perihematomal proportion of white matter survival positively correlates with hematoma volume and hemolysis volume at \&lt day 3 , day $14 \&$ day 30 .

Conclusion Our preliminary study suggests that MRI can reliably assess the proportion of erythrolysis that occurs within the hematoma. Our study also shows that there is reliability in measurement of tissue toxicity markers like iron that leaches out from the hematoma into surrounding tissue (IO) and its correlation with proportional lysis within the hematoma over a period of 1 month. In addition, hemolysis/eryhtrolysis percentage within hematoma and its size correlates with surviving white matter tissue around the hematoma. The above mentioned parameters could become objective markers of tissue injury secondary to an $\mathrm{ICH}$ and once validated further in a larger human sample could further inform the assessment of multiparametric prognostic indices of human ICH on MRI.

Disclosures N. Novakovic: None. Z. Wilseck: None. T. Chenevert: None. G. Xi: None. R. Keep: None. A. Pandey: None. N. Chaudhary: None.

\section{E-087 NEUROFORM ATLAS STENT-ASSISTED COILING: JAILING VS RE-CROSSING TECHNIQUES}

${ }^{1}$ A Khaldi ${ }^{*},{ }^{1} \mathrm{P}$ Williams, ${ }^{2} \mathrm{~A}$ Jadhav, ${ }^{3} \mathrm{R}$ Hanel, ${ }^{4} \mathrm{D}$ Frei, ${ }^{5} \mathrm{~S}$ Hetts, ${ }^{6} \mathrm{O}$ Zaidat, ${ }^{7} \mathrm{~B}$ Jankowitz. ${ }^{1}$ Neurosurgery, Wellstar Medical Group, Marietta, GA; ${ }^{2}$ Division of Neurological Surgery, Barrow Neurological Institue, Phoenix, AZ; ${ }^{3}$ Neurosurgery, Baptist Neurological Institute, Jacksonville, FL; ${ }^{4}$ Radiology Imaging Associates, Swedish Medical Center, Englewood, CO ${ }^{5}$ Interventional Neuroradiology, University of California San Francisco, San Francisco, $C A_{i}$ ${ }^{6}$ Neuroscience, Bon Secours Mercy, Toledo, OH; ${ }^{7}$ Neurosurgery, Cooper University Hospital, Camden, NJ

\subsection{6/neurintsurg-2021-SNIS.182}

Introduction Stent-assisted coil embolization of cerebral aneurysms can be performed using one of multiple techniques. Jailing is the ability to trap microcatheter in the aneurysm while laying a stent across the neck of the aneurysm followed by coiling the aneurysm. Crossing technique refers to placing a microcatheter through the interstices of the stent and then into the aneurysm in order to treat it. Neuroform Atlas stent can be delivered via a 0.0165 inch inner diameter microcatheter which would allow for easier placement of two microcatheters simultaneously and hence a more efficient trapping technique.

Method Over 298 patients were treated in multiple centers with Neuroform Atlas Stent-assisted coiling of aneurysms. The treating physicians decided on the technique of treatment including jailing, crossing, or others (jailing followed by recrossing, stenting followed by re-crossing at a different time, or unknown). Patients' demographics, shape, dimension, and location of the aneurysm, as well as the number of coils and procedure duration were analyzed ad hoc. Intra-procedure complications of the different techniques were also recorded. In addition, a 12-month follow up angiogram (248/298) was performed, and Raymond score was evaluated by core laboratory.

Results Similar characteristics were observed between the two groups (jailing and re-crossing) in the shape, dimensions, and location of the aneurysms. There was also no increase in the number of coils used or duration of the procedure between the two different techniques. Procedures related adverse events and device related adverse events were similar between the 2 groups. A higher proportion of complete occlusion and a lower proportion of residual aneurysm (2.7\%) were observed in the jailing group, compared with the re-cross group (7.3\%). However, the difference is not statistically significant.

Conclusion Jailing technique might present an alternative to re-crossing the Neuroform Atlas Stent without a significant increase in complications and it may decrease the chance of aneurysm recurrence at 12 months.

Disclosures A. Khaldi: None. P. Williams: None. A. Jadhav: None. R. Hanel: 1; C; Medtonic, Stryker, Cerenovus, Microvention. 2; C; Stryker, Medtronic, Cerenovus, Microvention. 4; C; Neurvana, Elum, EndoStream, Three Rivers Medical Inc, Cerebrotech, In NeuroCO.. 6; C; Scientific Advisor, MIVI, Scientific Advisor, Elum, Scientific Advisor, Three Rivers Medical. D. Frei: 2; C; Stryker, Siemens. 3; C; Stryker, Penumbra, Philips, Genetech, Siemens, VIZ AI. 6; C; Medical Advisor for Memory Medical. S. Hetts: 2; C; Microvention. 6; C; Core Lab services for Stryker and Micro Vention Terumo. O. Zaidat: 1; C; Stryker, Medtronic, Cerenovus, Penumbra, Genetech. 2; C; Cerenovus, Stryker, Penumbra, Medtronic. 6; C; Ownership in Galaxy Therapeutics LLC, Endovascular Committee co chair of NIH StrokeNet Consortium. B. Jankowitz: 2; C; Stryker, Medtronic.

\section{E-088 VISUALIZATION OF THE ANTERIOR TEMPORAL ARTERY AS A PREDICTOR OF OUTCOME AFTER MECHANICAL THROMBECTOMY}

${ }^{1} \mathrm{~J}$ Parish*, ${ }^{2} \mathrm{~W}$ Stetler, ${ }^{3} \mathrm{D}$ Strong, ${ }^{3} \mathrm{~T}$ Prasad, ${ }^{3} \mathrm{~J}$ Rhoten, ${ }^{4} \mathrm{R}$ Karamchandani, ${ }^{5} \mathrm{~J}$ Clemente, ${ }^{5} \mathrm{G}$ Defilipp, ${ }^{5} \mathrm{~A}$ Hines, ${ }^{2} \mathrm{~J}$ Bernard, ${ }^{3} \mathrm{~A}$ Asimos. ${ }^{1}$ Neurological Surgery, Atrium Health, Charlotte, NC; ${ }^{2}$ Carolina Neurosurgery and Spine Associates, Charlotte, NC; ${ }^{3}$ Atrium Health, Charlotte, NC; ${ }^{4}$ Neurology, Atrium Health, Charlotte, NC; ${ }^{5}$ Charlotte Radiology, Charlotte, NC

\subsection{6/neurintsurg-2021-SNIS.183}

Introduction The anterior temporal artery (ATA) visualization on Computerized Tomography Angiography (CTA) has been previously associated with good outcomes in middle cerebral artery (MCA) occlusions, but not in the context of recanalization after mechanical thrombectomy. We hypothesized that independent functional outcome at 90 days would be greater for MCA occlusion patients with a visualized ATA on CTA who achieved Thrombolysis in Cerebral Infarction (TICI) 2b or greater recanalization.

Methods We conducted a retrospective cohort study of patients with acute ischemic stroke who underwent mechanical thrombectomy. A neuroradiologist blinded to patient outcomes confirmed the MCA as the most proximal site of occlusion on CTA and assessed for visualization of the ATA. TICI $2 \mathrm{~b}$ or greater revascularization scores were confirmed by neurointerventionalists blinded to patient outcomes. Estimates of the volume of ischemic core and penumbral regions from CT perfusion were calculated with the use of RAPID software (iSchemaView). Ninety-day mRS scores were obtained via telephone utilizing a structured questionnaire. 